\title{
ISCEV standard for clinical multifocal electroretinography (mfERG) (2011 edition)
}

\author{
Donald C. Hood - Michael Bach • Mitchell Brigell • David Keating • \\ Mineo Kondo · Jonathan S. Lyons • Michael F. Marmor • Daphne L. McCulloch • \\ Anja M. Palmowski-Wolfe - For the International Society For Clinical Electrophysiology of Vision
}

Received: 9 October 2011 / Accepted: 12 October 2011/Published online: 30 October 2011

(C) Springer-Verlag 2011

\begin{abstract}
The clinical multifocal electroretinogram (mfERG) is an electrophysiological test of local retinal function. With this technique, many local ERG responses are recorded quasi-simultaneously from the cone-driven retina under light-adapted conditions. This document, from the International Society for Clinical Electrophysiology of Vision (ISCEV: www.iscev.org), replaces the ISCEV guidelines for the mfERG published in 2007. Standards for performance of the basic clinical mfERG test with a stimulus array of 61 or 103 hexagons, as well as for reporting the results, are specified.
\end{abstract}

D. C. Hood $(\bowtie)$

Departments of Psychology and Ophthalmology,

Columbia University, New York, NY, USA

e-mail:dch3@columbia.edu

M. Bach

Univ.-Augenklinik, Freiburg, Germany

M. Brigell

Translational Medicine, Novartis Institutes for

Biomedical Research, Cambridge, MA, USA

D. Keating

Department of Ophthalmology, Gartnavel General

Hospital, Glasgow, UK

M. Kondo

Department of Ophthalmology, Mie University Graduate

School of Medicine, Tsu, Japan
Keywords Clinical guidelines $\cdot$ Electroretinogram · Multifocal electroretinogram
Abbreviations
CRT Cathode ray tube
ERG Electroretinogram
ISCEV International Society for Clinical
LCD Llectrophysiology of
mfERG Multifocal electroretinogram
PERG Pattern electroretinogram

\author{
J. S. Lyons \\ Washington Hospital Center Program in Ophthalmology, \\ Georgetown University, Silver Spring, MD, USA \\ M. F. Marmor \\ Byers Eye Institute at Stanford, Stanford University \\ School of Medicine, Palo Alto, CA, USA \\ D. L. McCulloch \\ Vision Sciences, Glasgow Caledonian University, \\ Glasgow, UK
A. M. Palmowski-Wolfe
Department of Ophthalmology, University of Basel,
Basel, Switzerland




\section{Introduction}

The electroretinogram (ERG) is a mass potential, which reflects the summed electrical activity of the retina. Full-field electroretinography is a well-established clinical technique for evaluating global retinal function [1]. The multifocal ERG (mfERG) technique was developed to provide a topographic measure of retinal electrophysiological activity. With this technique, many local ERG responses, typically 61 or 103 , are recorded from the cone-driven retina under lightadapted conditions. In 2003, the International Society for Clinical Electrophysiology of Vision (ISCEV) published guidelines for recording the mfERG [2] and these guidelines were revised in 2007 [3]. Both documents were guidelines, not standards, to allow for further research before standards were set.

This Standard, which supersedes earlier ISCEV Guidelines for multifocal electroretinography [3], defines minimum protocols for basic clinical mfERG recording and reporting. The details of this protocol are less prescriptive than other ISCEV standards. For example, a range of values is allowed for both the luminance of the stimuli and the filtering by the amplifiers. There are a number of reasons for this decision. The first is that the technology for producing the stimulus display is rapidly evolving, with resultant differences in stimulus parameters. Second, there are no data to support the superiority of any single variant in producing repeatable results or in sensitivity to retinal disease. Thus, any of these technologies can produce clinically acceptable basic mfERG responses. Finally, the mfERG is primarily used in the clinic to localize damage spatially, so that variations in the topographic array of signals are more important than absolute signal size. This Standard defines the limits within which stimuli and recording conditions should lie as well as standardized modes of display so that responses can be recognized and compared from different laboratories worldwide.

This Standard defines the conditions and procedures for a "basic mfERG." It is important to keep a number of caveats in mind. First, the mfERG test does not replace the full-field ERG test. If pan retinal damage or damage to the rod system is suspected, then the ISCEV full-field ERG protocol [1] should be followed. Second, obtaining useful and reproducible mfERG recordings requires all the care that is needed for successful full-field ERG recordings, plus additional requirements, as described below. Third, this Standard only covers the primary use of the mfERG, which is to identify damage to the retina up to and including the inner nuclear layer. Experienced users may wish to add other test protocols or modify procedures in order to optimize the test for certain clinical applications.

ISCEV has also published guidelines for calibration of electrophysiologic equipment [4], and standards for full-field ERGs [1], pattern ERGs [5], the electrooculogram [6], and visual evoked potentials [7]. This standard will be reviewed periodically to incorporate developments and reflect current practice.

\section{Description of multifocal electroretinography}

The mfERG technique is a method of recording local electrophysiologic responses from different regions of the retina. Electrical responses from the retina are recorded with a corneal electrode as in conventional, full-field ERG recording. However, the nature of the stimulus and the form of the analysis differ. These differences allow a topographic map of local ERG activity to be measured. For the basic mfERG described here, the retina is stimulated with an array of hexagonal elements, each of which has a $50 \%$ chance of being illuminated every time the frame changes (Fig. 1). Although the pattern appears to flicker randomly, each element follows the same pseudo-random sequence of illumination with the starting point displaced in time relative to other elements. By correlating the continuous ERG signal with the sequence of on- and off-phases of each element, each local ERG signal is calculated. Although these local ERG signals are referred to as mfERG responses, it is important to keep in mind that they are not direct electrical potentials from local regions of retina, but rather they are a mathematical extraction of the signal. Further, because the stimulation rate is rapid, the waveform of the local mfERG response can be influenced both by preceding and subsequent stimuli, as well as by the responses to light scattered on other retinal areas.

The typical waveform of the basic mfERG response (also called the first-order response or first-order kernel) is a biphasic wave with an initial negative deflection followed by a positive peak (Fig. 2). There is usually a second negative deflection after the 

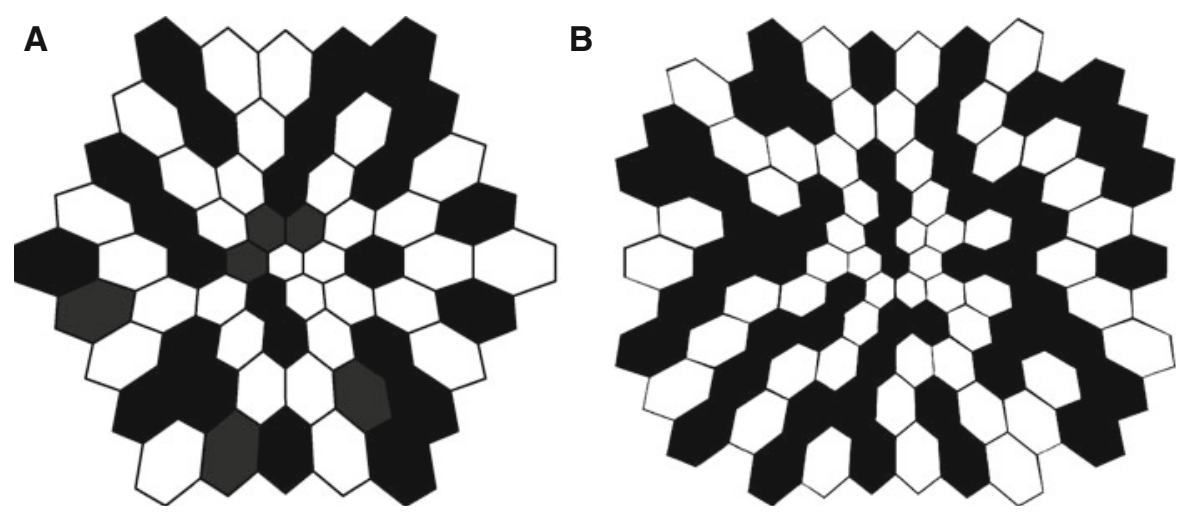

Fig. 1 a Representative hexagonal mfERG stimulus array with 61 elements scaled with eccentricity. Roughly half of the elements are illuminated at any one time. b Same as in panel $A$ for an array with 103 elements

positive peak. These three peaks are called N1, P1, and $\mathrm{N} 2$, respectively. There is evidence that $\mathrm{N} 1$ includes contributions from the same cells that contribute to the a-wave of the light-adapted, full-field ERG and that P1 and N2 include contributions from the cells contributing to the light-adapted b-wave and oscillatory potentials. Although there are homologies between the mfERG waveform and the conventional ERG, the stimulation rates are higher for the mfERG and, as noted above, the mfERG responses are mathematical extractions. Thus, technically the mfERG responses are not "low-amplitude ERGs". Therefore, the designations "a-wave" and "b-wave," used for full-field ERGs, are not appropriate to describe features of the mfERG waveform.

\section{Basic technology}

Electrodes

\section{Recording electrodes}

Electrodes that contact the cornea, or nearby bulbar conjunctiva, are required. This includes contact lens, foil, and fiber electrodes. In addition, good retinal image quality and proper refraction are desirable. The choice of electrode type can influence the signal-to-noise ratio (SNR) of the responses. For example, bipolar corneal contact electrodes typically yield recordings with the highest SNR. Thus, longer recording times, repeat measurements, and/or fewer stimulus elements are necessary to obtain comparable SNRs when using a foil or fiber electrode.

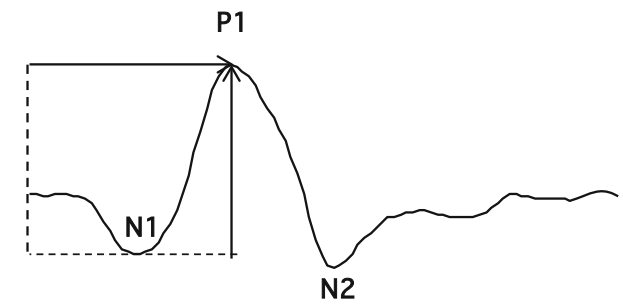

Fig. 2 Diagram of a mfERG response to show the designation of the major features of the waveform. The arrows show the trough-to-peak amplitude (vertical arrow) and the implicit time (horizontal arrow) of the basic mfERG measures of amplitude and timing

\section{Reference and ground electrodes}

Proper application of suitably conductive electrodes is essential for reliable mfERG recordings. Recordings are comparable only when the same electrode types and locations are used. Follow the recommendations made in other ISCEV Standards [1, 5].

\section{Electrode characteristics, stability, and cleaning}

Poor or unstable electrode contact is a major cause of poor-quality records. It is important to follow the recommendations concerning fiber, foil, loop, and contact lens electrodes in the full-field ERG Standard [1] and Pattern ERG (PERG) Standard [5].

\section{Stimulation}

\section{Stimulus source}

Until recently, mfERG stimuli were most commonly displayed on a cathode ray tube (CRT). CRT monitors 
are being rapidly replaced with other devices such as liquid crystal displays (LCDs). These alternative modes of stimulation can affect the amplitude and waveform of mfERGs making it essential to report the type of display and to specify the details of the manufacturer and model when reporting results. (See "Appendix B: Technical Details".)

\section{Frame frequency}

A CRT frame frequency of $75 \mathrm{~Hz}$ has been used widely, although $60 \mathrm{~Hz}$ has been used as well. Use of different frequencies can substantially alter the amplitude and waveform of the mfERG response. Whatever frame frequency is used, normative values for normal healthy subjects need to be determined separately for that frequency. It is essential to be aware of the frame frequency when interpreting results.

\section{Luminance and contrast}

For CRT displays, the luminance of the stimulus elements in the light state should be at least $100 \mathrm{~cd} / \mathrm{m}^{2}$. The luminance of the display in the dark state should be low enough to achieve a contrast (Michelson) of $\geq 90 \%$. With non-CRT displays (e.g., LCDs), the elements in the
A

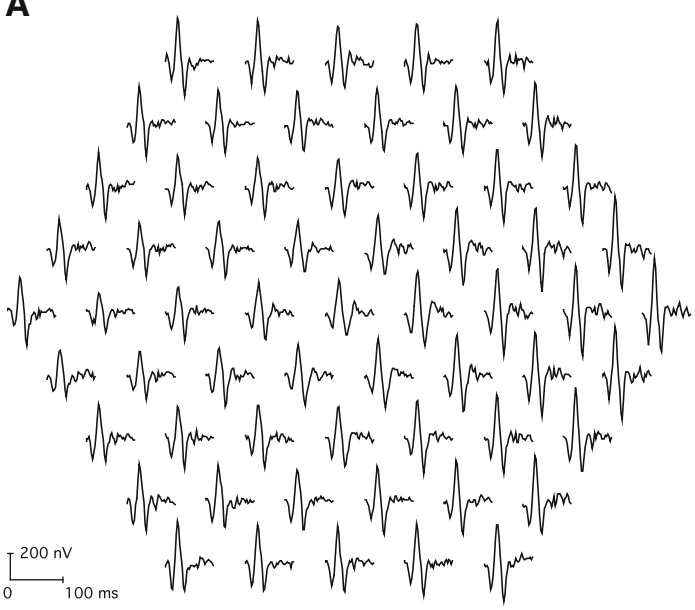

B

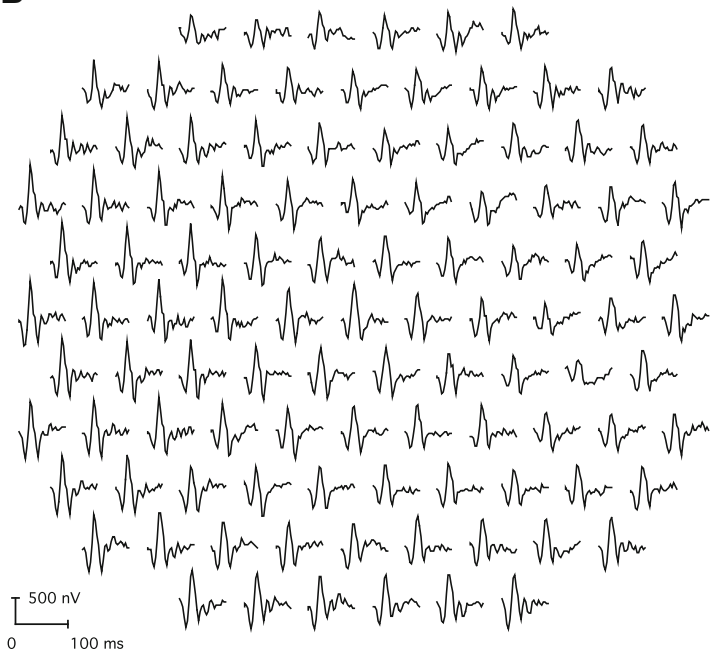

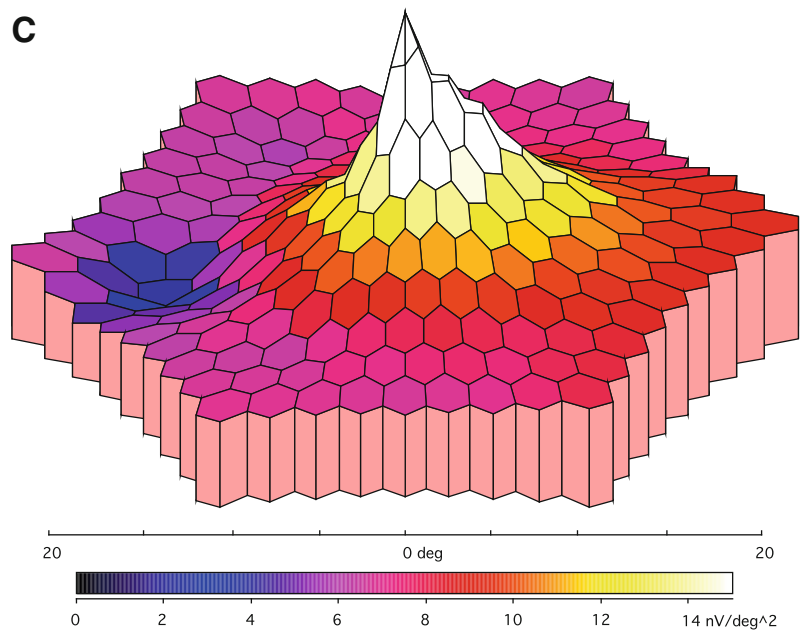

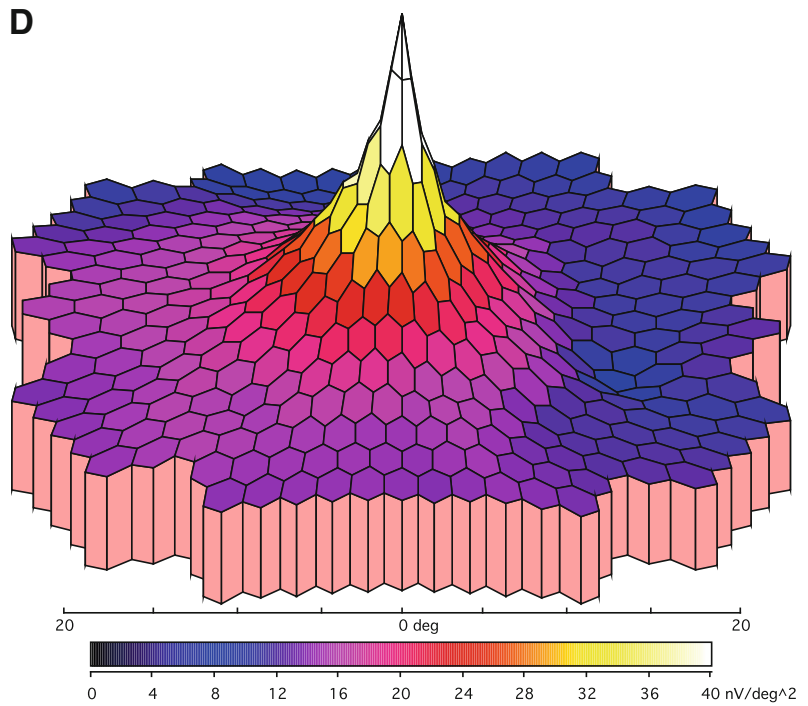

Fig. 3 Sample mfERG trace arrays (field view) with 61 elements (panel A, left eye) and 103 elements (panel B, right eye). c, d. The 3-D response density plots (field view) associated with panels $A$ and $B$ 


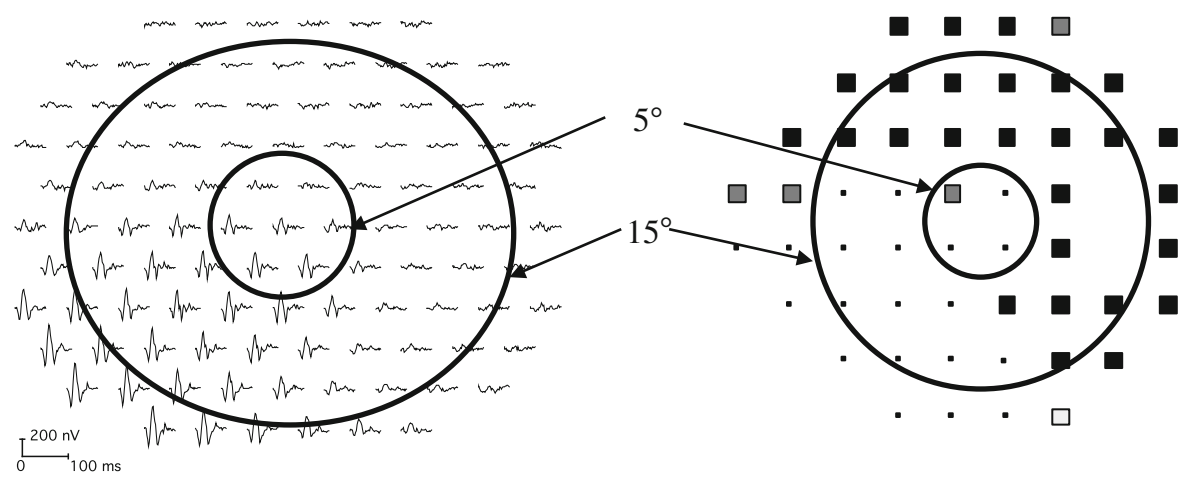

Fig. 4 The mfERG trace array (left panel, field view) and (right panel) the probability plot from routine automated perimetry (Humphrey Visual Field Analyser, Carl Zeiss Meditec) for a patient with retinitis pigmentosa. The contours for radii of 5 and

light state should be set to achieve reasonable mfERG amplitudes and clear waveforms as in Figs. 3 and 4; this may require higher luminance settings. With any display, higher luminance levels can be used if available, but the ability to detect local defects may be decreased due to the effects of stray light. For all standard recordings, the luminance of the surround region of the display (the area beyond the stimulus hexagons) should match the mean luminance of the stimulus array.

\section{Calibration}

As with other visual electrophysiologic tests, luminance and contrast affect the recorded signals and it is important for the stimulus to be calibrated following ISCEV guidelines [4]. The luminance of the dark and the light stimulus elements should be measured with an appropriate calibrator or spot meter. Many monitor screens are not of uniform brightness over the entire screen. While some variation is to be expected, a variation of greater than $15 \%$ is considered unacceptable. Some commercial systems are equipped to calibrate the display. If this ability is not present, manufacturers are urged to provide instructions for calibration of their devices.

Stimulus parameters

\section{Stimulus pattern}

The Standard display is a hexagonal stimulus pattern scaled in size to produce mfERG responses of $15^{\circ}$ are shown. The light gray, dark gray, and black squares indicate statistically significant field loss at the 5, 1, and 0.5 percent levels, respectively

approximately equal amplitude across the healthy retina. Thus, the central hexagons are smaller than the more peripheral ones. Different patterns may be useful in special cases (e.g., equal size hexagons for patients with eccentric fixation). However, this Standard covers only scaled hexagonal patterns.

Temporal sequence In mfERG testing, an algorithm (using an m-sequence) is used to control the temporal sequence of change between the light and dark stages of each stimulus hexagon. This m-sequence, in which each hexagonal element can change with every frame, is the standard for routine testing. Different sequences, or the inclusion of global light or dark frames, have been suggested for specialized applications, but these are non-standard, and should not be used in place of a "standard" mfERG for routine clinical purposes.

Stimulus size and number of elements The stimulus field consists of a hexagonal array with the fixation point at the center. For routine clinical examinations, the field should contain either 61 or 103 hexagons within a field diameter of $40^{\circ}-50^{\circ}\left(20^{\circ}-25^{\circ}\right.$ radius from the fixation point to edge of display); it is particularly important that the field is wide enough to include the blind spot. It is also important to specify, or ideally show on signal arrays, the dimensions of the stimulus zone in degrees so that comparisons can be made to fundus images and visual fields. The choice of 61 versus 103 elements depends on balancing the need for good spatial resolution and a high signal-to-noise ratio, while minimizing the recording time. Increasing the number of stimulus elements or decreasing the duration of the recording will decrease the signal-to-noise ratio of the responses. 
Decreasing the number of elements will increase the signal-to-noise ratio, but will decrease the spatial resolution of the test. While testing with fewer than 61 hexagons may be useful in some situations, such tests do not constitute a standard mfERG. In addition to a loss of spatial resolution, the quality of fixation will be more difficult to establish as the location of the blind spot may be difficult to indentify.

Fixation targets Stable fixation is essential for obtaining reliable mfERG recordings. Central fixation dots, crosses, and circles are available with most commercial systems. The fixation targets should cover as little of the central stimulus element as possible to avoid diminishing the response. However, the examiner should always verify that the patient can see the fixation target. When the fixation targets are enlarged for low-vision patients, care should be taken not to obscure regions of interest. For example, a larger central cross will lead to a smaller central response simply due to occlusion of more of the stimulus. If the patient cannot see the fixation target, the mfERG test will be of questionable value. Note that if the patient has good fixation in the other eye, the recording can be performed with both eyes open, although care is required in interpreting the results as there may be a misalignment between the two eyes (see "Monocular vs. binocular recording" below).

Recording and analysis

\section{Amplifiers and filters}

The gain of the amplifier should produce recognizable signals without saturation. Appropriate band-pass filtering removes extraneous electrical noise, without distorting waveforms of interest. The preprocessing filtering is accomplished by the amplifier and, in some cases, by the commercial software as well. For a basic mfERG, the band pass of the filters should be approximately 5-200 Hz. The acceptable range for the high pass cutoff is $3-10 \mathrm{~Hz}$ and for the low pass cutoff is 100-300 Hz. Filter settings, even within these ranges, can markedly influence the response waveform. Thus, the filter settings must be the same for all patients tested by a given laboratory, as well as for the norms to which they are compared. Line frequency or notch filters should be avoided. (See "Appendix B" for more details.)
Signal analysis

Artifact rejection Because blinks and other movements can distort the recorded waveforms, software algorithms for "artifact rejection" can eliminate some of these distortions. When applying an artifact rejection procedure after the recording, care should be exercised to assure that clinically important aspects of the waveform are not being modified. It is important to note that the artifact rejection algorithm used can affect the appearance of the resulting mfERG waveform. The methods used in the processing of the raw signals should be clearly stated by the manufacturers. (See "Appendix B" for more details.)

Spatial averaging To reduce noise and smooth waveforms, analysis software typically allows the averaging of the response from each stimulus element with a percentage of the signal from the neighboring elements. This spatial averaging can help visualize mfERG signals in noisy records. If used, the contribution from each neighbor should not exceed $17 \%$; at this level, the 6 neighbors of any hexagon contribute as much to the responses as does the local signal. Spatial averaging will obscure small, local changes or the borders of regions of dysfunction. Thus, it should be used with care and specified when reporting results. Further, the default conditions of commercial software should be examined and changed if necessary so that spatial averaging is not used as a default condition.

Signal extraction/kernels The standard response is the first-order kernel. Higher-order kernels, particularly the second-order kernel, are reported occasionally, and used in special applications.

\section{Clinical protocol}

Patient preparation

Pupils

The pupils should be fully dilated and pupil size noted.

\section{Patient positioning}

Patients should sit comfortably in front of the screen. Relaxation of facial and neck muscles will reduce 
artifacts from muscles; a chin and/or head-rest may be helpful. The pupil should be centered in the corneal electrode ring when using contact lens electrodes. The appropriate viewing distance will vary with screen size, in order to control the area (visual angle) of retina being stimulated.

\section{Fixation monitoring}

Good fixation, both central and steady, is essential. Thus, fixation should be monitored, preferably using monitoring instrumentation available on some units. When this option is not available, careful direct observation may be employed to assess the stability of fixation. The trace arrays and any 3-dimensional (3-D) plots for patients suspected of poor fixation due to reduced acuity or poor cooperation should be examined carefully for signs of eccentric fixation (see Fig. 7). Eccentric fixation can be compensated for by moving the fixation target.

\section{Refraction}

Although there is some evidence that mfERGs are unaffected by moderate blurring of the retinal image in healthy individuals, at least when within \pm 3 diopters, eyes should be refracted for optimal acuity at the viewing distance. On some commercial instruments, a manual adjustment of the viewing optics is possible. Alternatively, lenses can be placed in a holder positioned in front of the eye. In the latter case, care must be taken to avoid blocking the view of the stimulus screen by the rim of the lens or the lens holder and thus creating an apparent scotoma. It should be recognized that refractive correction also affects image size, an effect that becomes significant as the error increases. This is another reason to perform refractive correction, and it is important that the same correction be used for repeated testing of a patient to make results comparable.

\section{Monocular versus binocular recording}

Standard recording uses monocular stimulation. Binocular recordings should only be used if the eyes are aligned. Alignment may be difficult and ocular misalignment, such as may occur in a patient with a phoria, might only be manifested during the recording.

\section{Adaptation}

Pre-exposure to light The patient should be in ordinary room illumination before testing. Examinations such as indirect ophthalmoscopy and fundus photography should be performed after the mfERG procedure is completed. If such examinations have been performed before the mfERG, then a recovery time of at least $15 \mathrm{~min}$ is required. As near as is practical, the pre-test light exposure should be the same for all mfERG tests.

Room illumination Moderate or dim room lights should be on and ideally should produce illumination close to that of the stimulus screen. Ambient lighting should be the same for all recordings and care should be taken to eliminate any reflections from lens surfaces and to keep any bright light sources out of the patient's direct view.

Stimulus and recording parameters

\section{Stimulus size and number of elements}

A display that contains 61 or 103 elements subtending a total of $40^{\circ}-50^{\circ}$ (radius of $20^{\circ}-25^{\circ}$ to edge of display) with a central fixation point should be used.

\section{Duration of recording}

A total recording time of at least 4 min for 61 element arrays, or $8 \mathrm{~min}$ for 103 element arrays, is recommended, although experienced laboratories might adjust recording times to achieve stable waveforms. The overall recording time is divided into shorter segments (e.g., 15-30 s) so that the patient can rest between runs if necessary and also so that a poor record (from noise, movement, or other artifacts) can be discarded and repeated without losing prior data.

\section{mfERG interpretation and reporting}

Interpreting mfERG's is a two-step process. The first is to carefully inspect the waveforms in the trace array looking for variations in amplitude based on available clinical information (e.g., patient's complaints, visual fields). The second is to look at numeric data that show amplitudes relative to norms within averaged groups of responses. The primary clinical use of the mfERG is to detect spatial variations in mfERG responses that 
localize retinal damage to discrete regions of retina: the macula, paramacula, or discrete peripheral areas. Central retinal dysfunction will be evident as a loss of signal within the central regions of the mfERG; peripheral dysfunction with foveal preservation (as in retinitis pigmentosa) will be evident as strong central responses with weak or flat signals in the peripheral rings. Bull's eye maculopathy (e.g., in chloroquine retinopathy) will be evident as loss that is greatest in the parafoveal rings. And focal damage (e.g., infarction or scattered diabetic damage) will show as pockets of low amplitude or flat responses corresponding to the areas of disease. These clinical evaluations require examination of the trace array to recognize where signals are reduced in amplitude or delayed in timing relative to others.

The evaluation of "normal" responses is more complex for the mfERG than for the full-field ERG where normative confidence limits are used. In the case of the mfERG array, a sufficiently large normative database is often not available and statistical comparisons would involve many individual waveforms. Thus, recognition of "normal" mfERGs is based on the appearance of uniform well-defined waveforms with amplitudes typical for the individual laboratory. However, normative data are readily available or easily established for ring response plots described below. By looking at these numbers, the amplitude of individual responses relative to normal can be assessed (taking into account, of course, any focal loss within the ring).

In summary: (1) Critically examine the trace array for any areas of relatively small or delayed signals. (2) Make a judgment about the overall normality or abnormality of responses by taking into account the response values relative to norms and relative to the waveforms. 3-D representations and ring response plots (see below) may be helpful to identify potential areas of damage.

\section{Displaying results}

One of the important goals of standardization is that data are displayed in a comparable manner across centers, so that records are universally recognizable and interpretable. This means that certain basic display conventions are used and that critical information such as the mode of display (field view or retinal view) and the dimensions of the stimulus field (degrees) are provided in a readily visible fashion. Reports should include other critical information such as the equipment manufacturer, type of recording electrode, and comments on any issues affecting the recording (fixation quality, etc.).

\section{Trace arrays}

The mfERG is displayed as an array of the mfERG traces (Fig. 4a, b). The trace array is the basic mfERG display and should always be included in the report of clinical results. In addition to showing topographic variations, these arrays also demonstrate the quality of the records, which is important in judging the validity of any suspected variations from normal. Note that the topographical representation in Fig. 3 is only approximate, as the central responses would overlap if centered on the hexagons in Fig. 1. Trace lengths of $100 \mathrm{~ms}$ or more should be used for these displays. (It is hard to detect interference from line frequency and/or kernel overlap in shorter trace lengths.) Further, the trace array can be displayed with either a visual field view or retinal view. "Field view" or "retinal view" should be specified on the array, along with notation of the breadth (approximate width in degrees) of the trace array.

Some laboratories have developed displays to directly compare mfERG trace arrays to visual fields or to fundus images. These can be very useful clinically, but are not standardized displays. Figure 4 illustrates how a spatial comparison to visual field test results can help to confirm the relation between visual field defects and retinal dysfunction.

\section{Topographic (3-D) response density plots}

The 3-D plot (Fig. 3c, d) shows the overall signal strength per unit area of retina. This display can be useful for assessing the quality of fixation by observing the location and depth of the blind spot. In general, if fixation is steady and central, a clear depression due to the blind spot should be present and located in the appropriate place. That is, the presence of a blind spot assures that fixation was good. The absence of a blind spot can be due to poor fixation or a generalized loss of signal due to disease.

However, there are major dangers involved in using the 3-D plot to assess retinal damage. First, information about the waveforms is lost. Thus, large, 
but abnormal, or delayed responses can produce normal 3-D plots. Second, a central peak in the 3-D plot can be seen in some records without any retinal signal (see, "Appendix A: Artifact recognition examples for line frequency and weak signals"). Finally, the appearance of the 3-D plot from a given recording is dependent on how the local amplitude is measured. For these reasons, 3-D plots should not be used without simultaneous display of the trace array.

\section{Ring and other regional averages}

Groups of responses from the trace arrays can be averaged to compare quadrants, hemiretinal areas, normal and abnormal regions of two eyes, or successive rings from center to periphery. In addition, responses from stimulus hexagons associated with a local area of interest can be averaged for comparison with a similar area in an unaffected eye or with data from control subjects. Averaging responses within rings around fixation are particularly useful when studying patients with diseases that produce dysfunction with approximate radial symmetry. Figure 5 illustrates two ways to average the responses within a ring for a 61-hexagon array (see inset). First, to obtain the average response per hexagon, the responses from the hexagons in each ring are summed and then this summed response is divided by the number of hexagons in the ring. This average response/hexagon is shown in Fig. 5a. It will be roughly constant across rings because the stimulus hexagons are scaled to provide approximately equal response amplitudes. However, this scaling will not be perfect for all individuals or display sizes.

Figure $5 \mathrm{~b}$ illustrates a second useful way to display the ring responses. Recall that the hexagons are not all the same size; they are scaled with eccentricity (see Fig. 1). One can display the responses within a ring as amplitude/unit area (i.e., response density). In Fig. 5b, the sum of the responses in each ring is divided by the total area of the hexagons in the ring and plotted as $\mathrm{nV} /$ $\mathrm{deg}^{2}$. The response is largest in the center (fovea) where the cone photoreceptors and bipolar cells are densest. Both types of ring display allow measurement of P1-N1 amplitude and latency, which can be compared to normative data (see below) for judging the general size and timing of signals in a given patient. Some have found that the ratios of these ring values to one another are of use in detecting abnormalities such as parafoveal loss, because the ratios between rings are a relatively stable value across normals. However, clinics will need to establish norms
A

Response/Hexagon

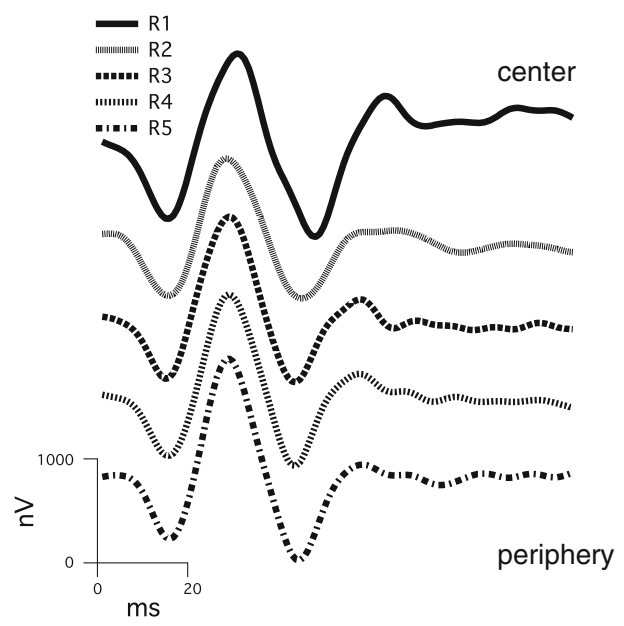

Fig. 5 a The mfERG responses in Fig. $3 b$ were grouped by concentric rings (see inset in the middle) and the average response per hexagon within each ring was calculated. That is, the sum of all the responses in a ring is divided by the number of the hexagons in the ring to give the average response per
B

Response/Degree ${ }^{2}$

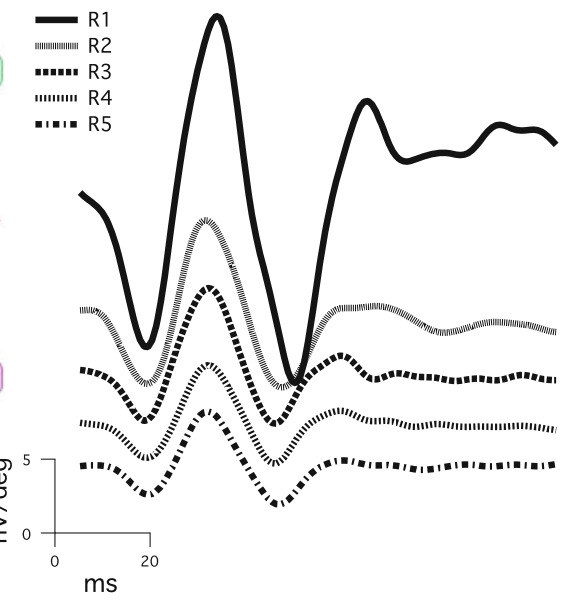

hexagon in $\mathrm{nV}$. $\mathbf{b}$ The sum of the responses in a ring is divided by the area of the hexagons in the ring to give $n V /$ degree $^{2} . R 1$ is central; $R 5$ peripheral. A similar grouping for the 103 hexagon display would have 6 rings 
for the ratios they wish to use. If ring ratios are used, it is best not to use ring 1 as the only reference as it is the most variable among normal controls.

\section{Measurements and calibration marks}

Calibration marks must accompany all traces or graphs. This will enable comparisons among patients or within a patient on sequential visits.

\section{Measuring mfERG amplitude and timing}

The standard measurement for mfERG amplitude is the trough-to-peak amplitude, measured from the trough of $\mathrm{N} 1$ to the peak of $\mathrm{P} 1$ and shown as the vertical arrow in Fig. 2. The standard measurement for timing is the implicit time of P1 (horizontal arrow in Fig. 2), i.e., the time at which the peak of $\mathrm{P} 1$ occurs. In some cases, the amplitude and timing of N1 may be of interest, but these measurements are not part of this Standard.

Commercial software typically provides measures of the overall amplitude and timing of the mfERG traces. There are various procedures for measuring amplitude (e.g., trough-to-peak amplitude), latency (e.g., response shifting, response stretching, time to peak), or overall response waveform (e.g., scalar product, root-mean-square (RMS)). A description of these techniques is beyond the scope of this standard. However, it should be noted that when a template is needed (e.g., for scalar product measures), the template should be formed from age-similar control data obtained from that laboratory.

\section{Normal values}

When normative data are used, each laboratory must develop its own database. Variations in recording equipment and parameters make the use of data from other sources inappropriate. Because electrophysiologic data are not necessarily described by a normal distribution, laboratories should report median values rather than means and determine boundaries of normality. The mfERG, like the full-field ERG, is smaller in amplitude in older individuals and in those with highly myopic eyes so that age and refractive error may be important in the evaluation of some patients. Thus, age-adjusted normative data are recommended.

\section{Reporting of artifacts and their resolution}

Reports should indicate any problems with the recording such as eye movements, head tilt, poor fixation, media opacities, poor refractive correction, etc. that might affect reliability and interpretation.

Acknowledgements This document was approved by ISCEV, September 22, 2010 at the Annual General Meeting in Lac Beauport, Quebéc, Canada. This document is a free publication in Documenta Ophthalmologica (without subscription) and can be accessed through links on the ISCEV website: http://www.iscev.org.

Conflict of interest Dr Keating has received funding for consultancy from Scottish Health Innovations Limited, an organisation responsible for commercialising Intellectual Property which originates in NHS Scotland.

\section{Appendix A: artifacts in mfERG recordings}

There are a number of artifacts that can affect the recording or interpretation of mfERGs. Some of the more common ones are mentioned below, along with brief suggestions for the avoidance and/or correction of these problems.

Common types of artifact

\section{Line frequency interference (Fig. 6)}

Poor electrode contacts, poor grounding, or ambient sources of noise can cause line current $(50$ or $60 \mathrm{~Hz})$

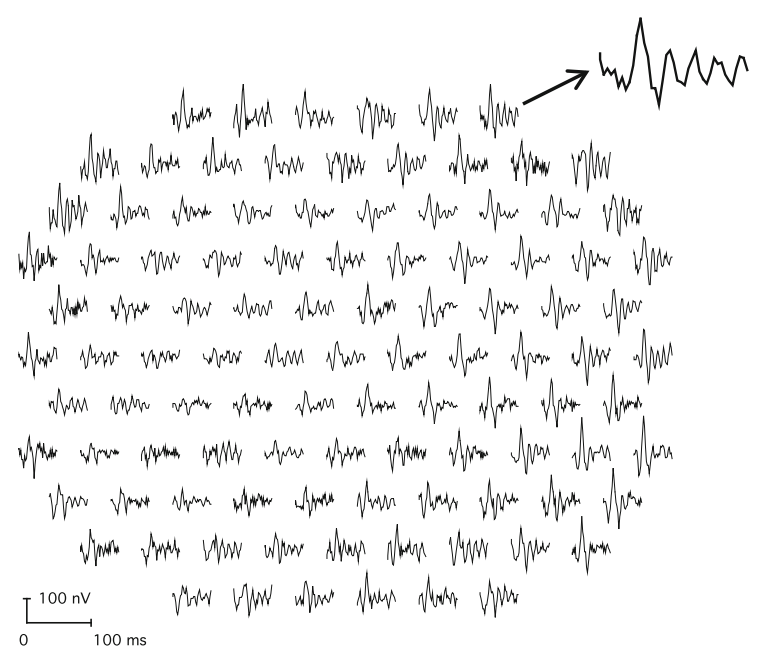

Fig. 6 Electrical noise. The trace array shows $60 \mathrm{~Hz}$ line frequency contaminating the responses 
interference that alters the mfERG traces. Noise is usually easy to identify in trace arrays if the time epoch of the trace is sufficiently long. Solution: Better electrode contact, grounding, and/or electrical shielding. While this line frequency noise can be removed after the recording using narrow digital band-pass filters, it is not the preferred solution. Line interference should not occur under optimal conditions and the presence of high amplitude line interference may indicate poor recording conditions (e.g., poor electrode contact) which may make results invalid.

\section{Movement errors}

Large eye movements can produce saturation of the amplifiers as well as aberrant drifting or fluctuation in the signal recorded. Milder degrees of eye movement, or unsteady fixation, can cause a smearing of the responses among different loci, and thus reduce the resolution, making it difficult to detect small areas of dysfunction. If the blind spot is not visible in the 3-D density plot, this may be a clue to poor fixation. Solution: Observe the amount of noise during the recording. Contaminated runs or segments should be discarded and re-recorded. The monitoring and control of fixation should be improved.

\section{Eccentric fixation (Fig. 7)}

Eccentric fixation can cause trace arrays and topographic 3-D plots that are depressed centrally, or show a "sloping" appearance with low signals on one side and high on the other. Solution: Check fixation and use a special low vision fixation target or displace the fixation target so that the fovea will correspond to the central hexagon.

\section{Positioning errors (Fig. 8)}

In some recordings from healthy eyes, the trace arrays and 3-D plots show depression in one part of the array and sometimes an elevation on the opposite side. This pattern can result if the refraction lens or the recording contact lens is blocking some of the display or if the patient is not perfectly aligned with the center of the lens when an optical system is interposed between the patient's eye and the screen. These errors must be distinguished from patterns of disease and from the small normal nasal-temporal variation. Solution: Center the lenses and patient; place the refracting lens close to the eye and monitor eye position.

\section{Erroneous central peak (Weak signal artifact) (Fig. 9)}

When using a display of scaled elements (Fig. 1), 3-D density plots can show a central peak, even when no signal is present. This occurs because the response amplitude (signal plus noise) is divided by the area of the hexagon. The effects of noise are magnified in the center where the overall amplitude is divided by a small area. Solution: Look at the trace array to determine whether any recognizable waveforms are present.
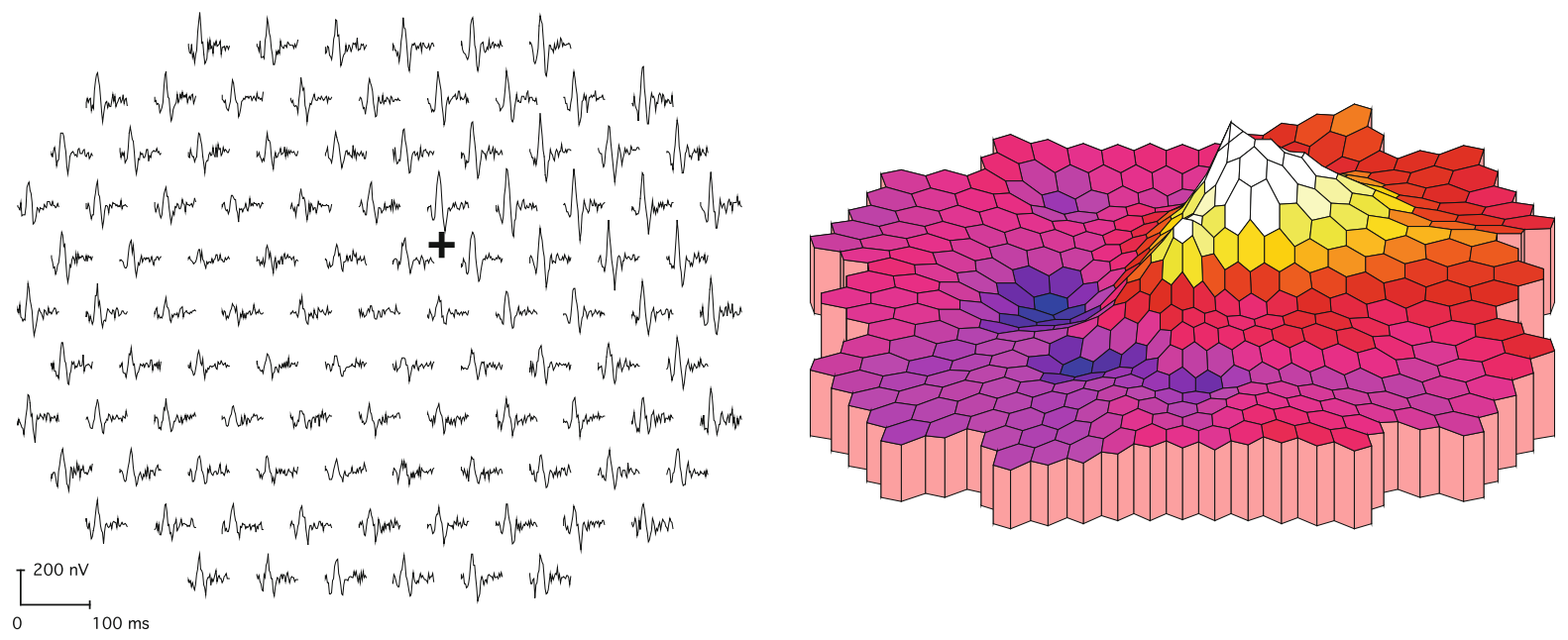

Fig. 7 Eccentric fixation. The subject with normal vision fixated at the + instead of at the center. As a result, the calculated response magnitudes are altered, and there is a false appearance of central retinal dysfunction 


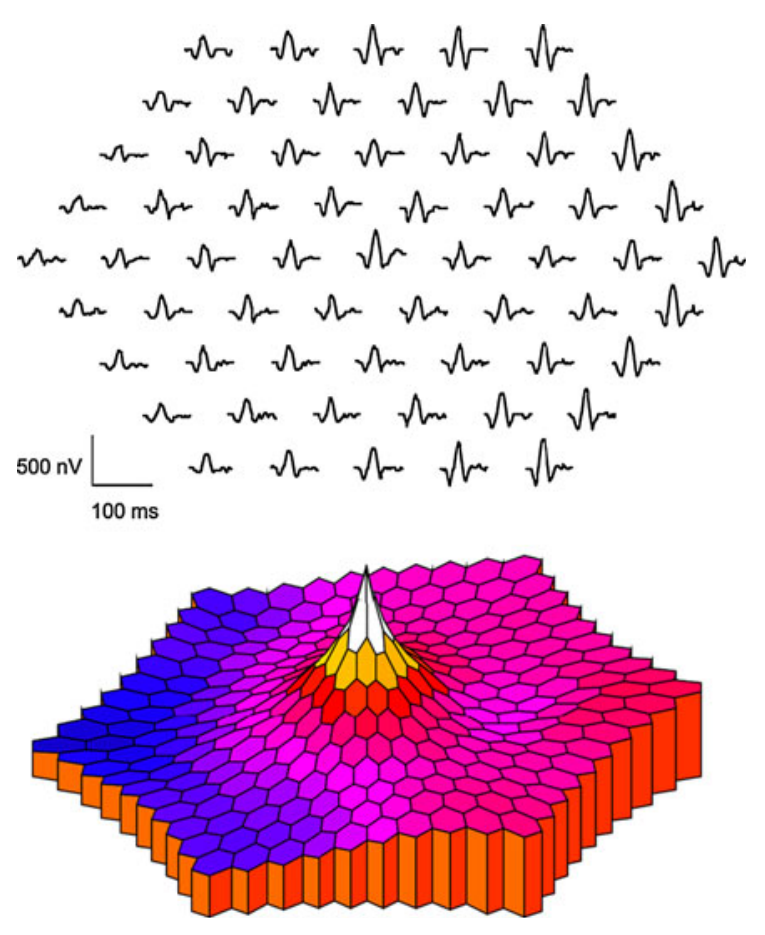

Fig. 8 Positioning error

\section{Averaging and smoothing artifacts}

Averaging or smoothing of signals will reduce spatial resolution. Severely smoothed records may not reveal small lesions, or show sharp lesion borders. Solution: Avoid unnecessary smoothing, and avoid excessive spatial averaging. Averaging beyond an equal contribution from all neighbors (17\% from each) is not recommended.

\section{Blind spot}

It is not an artifact that the blind spot is less sharply defined in the mfERG than one might expect. A single stimulus element may not fall totally within the optic disk, so that some response is always obtained. Further, the response attributed to an element falling anywhere in the field contains a contribution from neighboring regions receiving stray light scattered from this element. The nerve head may reflect more light than other areas of retina, and thus the stray light component associated with elements falling on the disk may be disproportionately large.

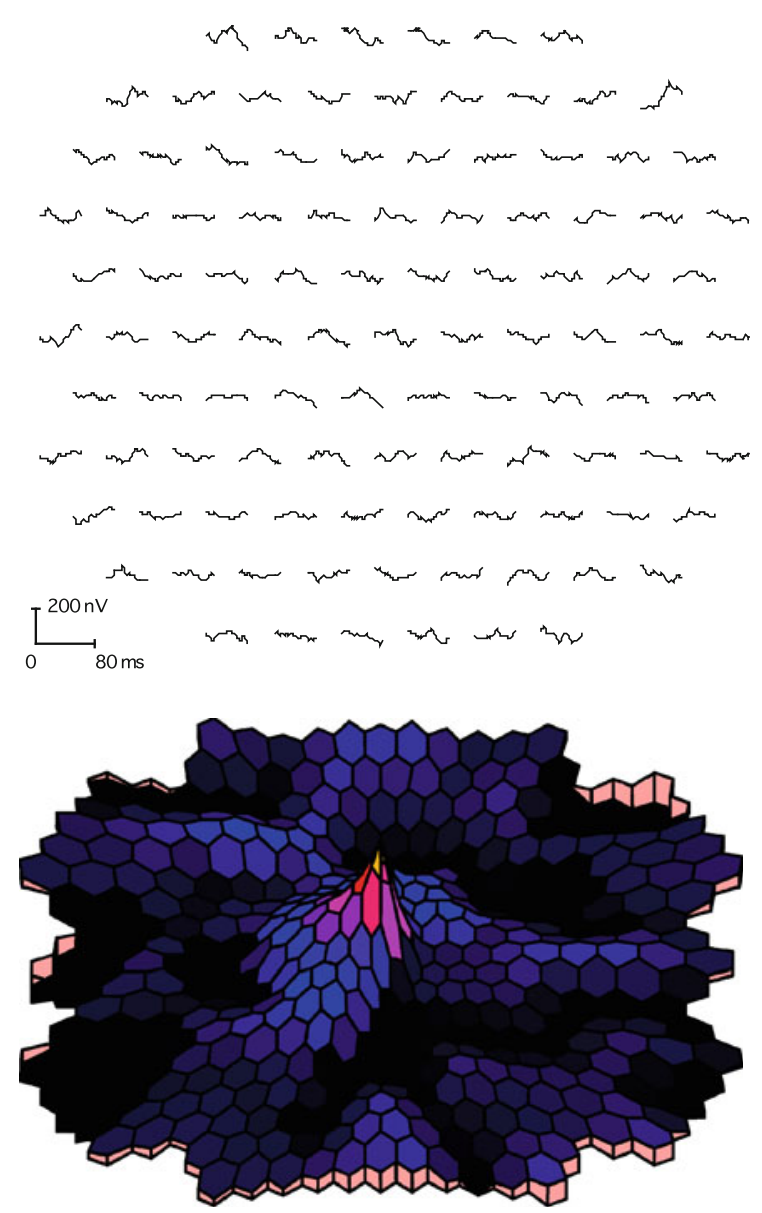

Fig. 9 Weak signals and erroneous central peak. These recordings were obtained from a contact lens electrode placed in a beaker of water. Therefore, there are no mfERG responses in these records. However, the 3-D plot shows an artifactual central peak because the noise level is divided by the stimulus area

\section{Appendix B: technical details}

This section contains technical details for manufacturers of mfERG equipment and for users wishing more detailed information.

\section{Response time of displays}

The term "response time" means the amount of time a local element (e.g., pixel) takes to go from "black" to "white" and back to "black" again. The response time must be sufficiently brief. This is not a problem with CRT monitors, which typically present a flash with a microsecond rise time followed by a $2 \mathrm{~ms}$ decay time at the beginning of each video frame. However, LCD 
panels typically switch between states and remain dark or bright for most of the frame. The switching times bright-dark versus dark-bright are not necessarily equal. The response times of some of the older LCD displays can be as long as $25 \mathrm{~ms}$. Response times should be considerably less than the frame interval (e.g., $\ll 13.33 \mathrm{~ms}$ for a frame rate of $75 \mathrm{~Hz}$ ). Manufacturers should supply information about the response time of non-CRT monitors or other display technology.

Filtering, artifact reject, and spatial averaging

Filtering can take place at a number of stages in the process from signal acquisition to the display of the responses. The amplifiers filter the input signal; the software may also allow for digital filtering before or after other processing; the artifact reject procedures can add filtering; and spatial averaging of signals will also alter the appearance of local responses. In setting default conditions for their software, manufacturers should take note of the standards set above. For example, line frequency filters and spatial averaging should not be set by default, and the default filter settings of the amplifier and software filters should conform to the standards for basic mfERG recording above. It is preferable if manipulations to reduce noise can be applied retrospectively so that the uncorrected data can be examined. In addition, because the artifact rejection algorithms can affect the waveform and amplitude of the responses, these procedures should be clearly specified either in the manufacturer's manual and/or a publication that is referenced.

\section{References}

1. Marmor MF, Fulton AB, Holder GE, Miyake Y, Brigell M, Bach M (2009) ISCEV standard for clinical electroretinography (2008 update). Doc Ophthalmol 118:69-77

2. Marmor MF, Hood D, Keating D, Kondo M, Seeliger MW, Miyake Y (2003) Guidelines for basic multifocal electroretinography (mfERG). Doc Ophthalmol 106:105-115

3. Hood DC, Bach M, Brigell M, Keating D, Kondo M, Lyons JS, Palmowski-Wolfe AM (2008) ISCEV guidelines for clinical multifocal electroretinography (2007 edition). Doc Ophthalmol 116:1-11

4. Brigell M, Bach M, Barber C, Moskowitz A, Robson J (2003) Guidelines for calibration of stimulus and recording parameters used in clinical electrophysiology of vision. Doc Ophthalmol 107:185-193

5. Holder GE, Brigell M, Hawlina M, Meigen T, Vaegan, Bach M (2007) Standard for pattern electroretinography. Doc Ophthalmol 114:111-116

6. Marmor MF, Brigell MG, McCulloch DL, Westall CA, Bach M (2011) ISCEV standard for clinical electro-oculography (2010 update). Doc Ophthalmol 122:1-7

7. Odom JV, Bach M, Brigell M, Holder G, McCulloch DL, Tormene AP, Vaegan (2010) ISCEV standard for clinical visual evoked potentials (2009 update). Doc Ophthalmol 120:111-119 